ARTICLE

\title{
Genome-wide association studies provide insights into the genetic determination of fruit traits of pear
}

Ming-Yue Zhang ${ }^{1,7}$, Cheng Xue (1) 1,2,7, Hongju Hu ${ }^{3,7}$, Jiaming $\mathrm{Li}^{1,7}$, Yongsong Xue ${ }^{1,7}$, Runze Wang ${ }^{1}$, Jing Fan ${ }^{3}$, Cheng Zou ${ }^{4}$, Shutian Tao', Mengfan Qin', Bing Bai ${ }^{1}$, Xiaolong $\mathrm{Li}^{1}$, Chao Gu${ }^{1}$, Shan Wu ${ }^{5}{ }^{5}$, Xu Chen²,

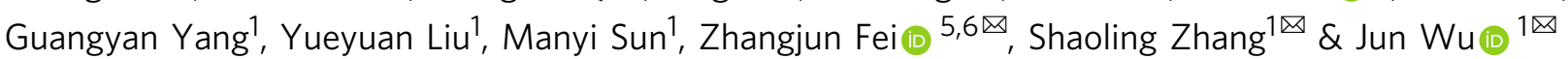

Pear is a major fruit tree crop distributed worldwide, yet its breeding is a very timeconsuming process. To facilitate molecular breeding and gene identification, here we have performed genome-wide association studies (GWAS) on eleven fruit traits. We identify 37 loci associated with eight fruit quality traits and five loci associated with three fruit phenological traits. Scans for selective sweeps indicate that traits including fruit stone cell content, organic acid and sugar contents might have been under continuous selection during breeding improvement. One candidate gene, PbrSTONE, identified in GWAS, has been functionally verified to be involved in the regulation of stone cell formation, one of the most important fruit quality traits in pear. Our study provides insights into the complex fruit related biology and identifies genes controlling important traits in pear through GWAS, which extends the genetic resources and basis for facilitating molecular breeding in perennial trees.

\footnotetext{
${ }^{1}$ Centre of Pear Engineering Technology Research, State Key Laboratory of Crop Genetics and Germplasm Enhancement, Nanjing Agricultural University, Nanjing Jiangsu, China. ${ }^{2}$ Haixia Institute of Science and Technology, Fujian Agriculture and Forestry University, Fuzhou, China. ${ }^{3}$ Wuchang Sand Pear Germplasm, Hubei Academy of Agricultural Sciences, Wuhan, Hubei, China. ${ }^{4}$ Institute of Crop Sciences, Chinese Academy of Agricultural Sciences, Beijing, China. ${ }^{5}$ Boyce Thompson Institute, Cornell University, Ithaca, NY, USA. ${ }^{6}$ USDA-ARS, Robert W. Holley Center for Agriculture and Health, Ithaca, NY, USA. ${ }^{7}$ These authors contributed equally: Ming-Yue Zhang, Cheng Xue, Hongju Hu, Jiaming Li, Yongsong Xue. ${ }^{凶}$ email: zf25@cornell.edu; slzhang@njau.edu.cn; wujun@njau.edu.cn
} 
ear has been cultivated by human for at least 3000 years ${ }^{1}$. However, traditional breeding procedure of pear normally takes at least 13-15 years because of its long juvenile stage. Molecular breeding is an efficient way to help improve cultivars using pre-selection from large numbers of diverse individuals to identify and introduce precise genetic regions that can be expected to confer desired phenotypic benefits. For example, previous work identified 31 quantitative trait loci (QTLs) for 11 traits based on a pear linkage map constructed with 3241 markers $^{2}$. Amplified fragment length polymorphism (AFLP) markers were identified to be linked to a scab resistance gene in an interspecific hybrid pear ${ }^{3}$, and fire-blight resistance-related QTLs were identified in European pears ${ }^{4,5}$. Pear harvest time and skin colorrelated QTLs were also investigated in Japanese pears ${ }^{6}$. However, pear research and breeding have been limited by factors such as the time-consuming process of constructing cross populations for genetic mapping, and restricted genetic diversity.

With the completion of pear whole-genome sequencing, analyzing large natural populations through high-throughput sequencing and genome-wide association studies (GWAS) provides tremendous opportunities for exploring variation alleles and key genes underlying important traits, which can help to develop molecular breeding markers and reveal genetic mechanisms of these traits. In plants, GWAS was initially performed in cereal crops, such as maize, for which flowering time was found to be associated with Drawf8 polymorphisms ${ }^{7}$. In rice, GWAS was performed for 14 traits using a population consisting of 517 landraces with 3.6 million single nucleotide polymorphisms $(\mathrm{SNPs})^{8}$. GWAS was also performed in rice to rapidly identify genes influencing agronomic traits using the gene-based association analysis 9 . Despite the wide applications of GWAS in cereal and other annual crops, only a few GWAS investigations have been conducted for perennial fruit trees till now. One addressed 129 peach accessions and explored 12 fruit and flower-related traits ${ }^{10}$. A second study explored pathogen resistance in apricot ${ }^{11}$. Recently, a $200 \mathrm{~K}$ array was developed to facilitate GWAS of two traits in pear ${ }^{12}$. Therefore, additional GWAS with large population size, wide genetic background, and high-density SNPs are necessary to better understand the genetic architecture of fruit quality and phenological traits for fruit tree crops.

The skin color and the contents of sugars, acids, and stone cells are important pear fruit quality traits and also the main targets for pear fruit improvement. However, studies of fruit quality traits in pears are relatively limited compared with other fruit trees such as apple and grape, with only a few reports on pear fruit skin color $^{13}$, sugar ${ }^{14}$, organic acid ${ }^{15}$, and stone cells ${ }^{16,17}$. Pear fruits have special brachysclereid cells called stone cells with secondary-thickened cell walls that are formed from parenchyma cells via the deposition of lignins and celluloses ${ }^{18}$. Recently it was determined that the lignin forms present in pear stone cells are primarily guaiacyl-lignin (G-lignin) and syringyl-lignin (S-lignin) monomers ${ }^{19}$.

In this study, we carry out whole-genome resequencing with an average of around ten-fold coverage on an association panel comprising 312 sand pear (Pyrus pyrifolia) accessions collected from China, Korea, and Japan, and revealed their population structure. Meanwhile, landrace and cultivated sand pears are explored to identify selective sweep signatures in the genome during pear improvement. We then perform GWAS of 11 agronomic traits for which phenotype data are collected over three successive years, to identify the associated genome loci and candidate functional genes. A gene involved in regulating lignin contents is identified to function in stone cell formation in pear fruits, and it is verified in both transgenic pear and Arabidopsis. Genome loci associated with agronomic traits of pear and the identified gene involved in pear stone cell formation provide valuable information for fruit trees breeding, and for deeper understanding of the fruit biology.

\section{Results and discussion}

Sequencing, variants, and population structure of sand pears. We analyzed a germplasm diversity panel comprising 312 sand pears ( $P$. pyrifolia), of which 231 were landraces and 81 were improved pear cultivars (Supplementary Data 1). Genomes of these accessions were sequenced using the Illumina technology, and a total of $2.15 \mathrm{~Tb}$ of raw sequence data were obtained. After removing low-quality and adapter sequences, an average of about ten-fold coverage data were obtained for each accession and used for SNP calling. The average mapping rate of the cleaned reads to the reference genome of Pyrus bretschneideri ${ }^{20}$ was $73.5 \%$ (Supplementary Data 1), higher than that in a previous study of 113 pears from different species $^{21}$.

A total of 3.40 million SNPs with missing rate of no more than $30 \%$ and a minor allele frequency $(\mathrm{MAF}) \geq 0.03$ were obtained and used for downstream analyses. We randomly selected 3942 SNP loci for Sanger sequencing, which revealed a high accuracy of the called SNPs (97.6\%; Supplementary Data 2). Using these high-quality SNPs, we first inferred phylogenetic relationships of the sand pears, which suggested that the sand pears we analyzed were divided into two major subpopulations, one comprising accessions from China (Group I) and the other comprising accessions from Japan and the Korean peninsula (Group II) (Fig. 1a). This was largely consistent with the results obtained from the population structure and $F_{\mathrm{ST}}$ analysis (Fig. $1 \mathrm{~b}$ and Supplementary Figs. 1-3) and the principal component analysis (PCA) (Fig. 1c).

Selective sweep signals during pear improvement. The nucleotide diversity $(\pi)$ of the entire sand pear population here was $1.73 \times 10^{-3}$, and that of the landrace $\left(1.75 \times 10^{-3}\right)$ was higher than that of the improved pears $\left(1.70 \times 10^{-3}\right)$ (Fig. 2a). As expected, the diversity level we detected here for sand pear was substantially lower compared to that $\left(5.5 \times 10^{-3}\right)$ of combined Asian and European cultivated and wild pears reported previously $^{21}$. This previous study ${ }^{21}$ indicated that similar diversity was preserved in pears during the domestication process, which is different from the strong selection in annual crops, and during the improvement process, the diversity reduction in pear was slightly lower than that in annual crops such as soybean and rice 22,23 . At the transcriptome level, $\pi$ of pear landrace group was also higher than that in the improved group, but $\pi$ remains the same level from wild group to landrace group ${ }^{24}$. The linkage disequilibrium (LD) decay of the improved pears was less rapid than that of landraces (Fig. 2b). What was more, the LD decay in pears was much more rapid than that in annual crops like rice ${ }^{23}$ and soybean ${ }^{25}$, but similar to that in apple 26 , mainly due to the long generation time and self-incompatibility.

We identified candidate selective sweeps during pear improvement based on composite likelihood rates and nucleotide diversity throughout the genome for all of the accessions in the germplasm diversity panel of sand pears, which in total covered $11.1 \mathrm{Mb}$ (2.89\%; Supplementary Data 3) of the pear genome (Fig. 2c) and harbored 1417 genes (Supplementary Data 4), of which some were known to be related to metabolisms of sugars, organic acids, amino acids, lignin, as well as resistance and photosynthesis. We previously found that during the domestication process, genes related to metabolisms of sugars, organic acids, lignin were also under selection ${ }^{21}$. However, the fruit size-related genes were enriched in domestication sweeps ${ }^{21}$ while no such genes were found in selective sweep regions during pear improvement. We conclude that traits of lignin, sugar, and acid contents were under 

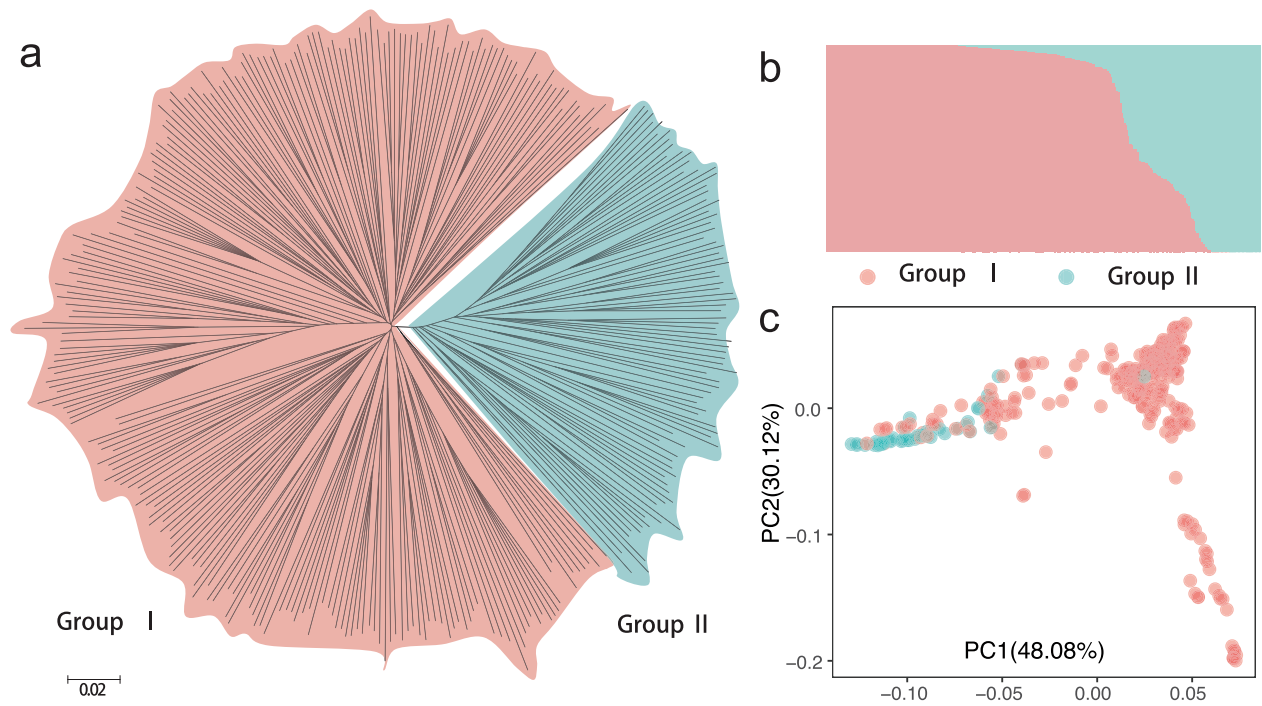

Fig. 1 Phylogenetic relationship and population structure of $\mathbf{3 1 2}$ sand pears. a Phylogenetic tree of sand pears. $\mathbf{b}$ Population structure of sand pears. $K$ value is 2. c PCA of sand pears. Group I with red background corresponds to the Chinese group, and Group II with blue background indicates the Japanese and Korea group.

a

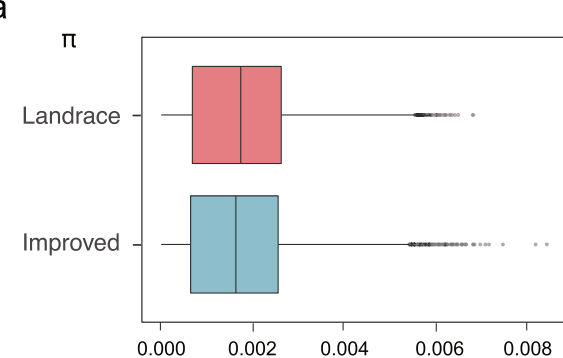

b

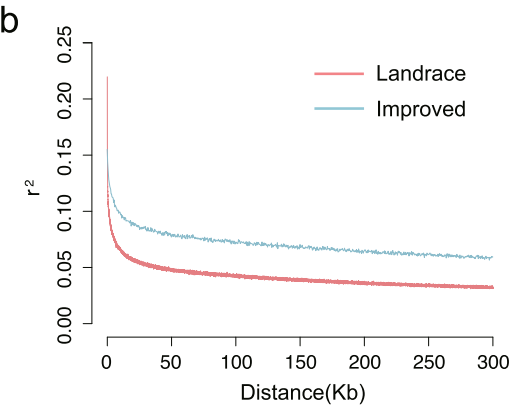

C
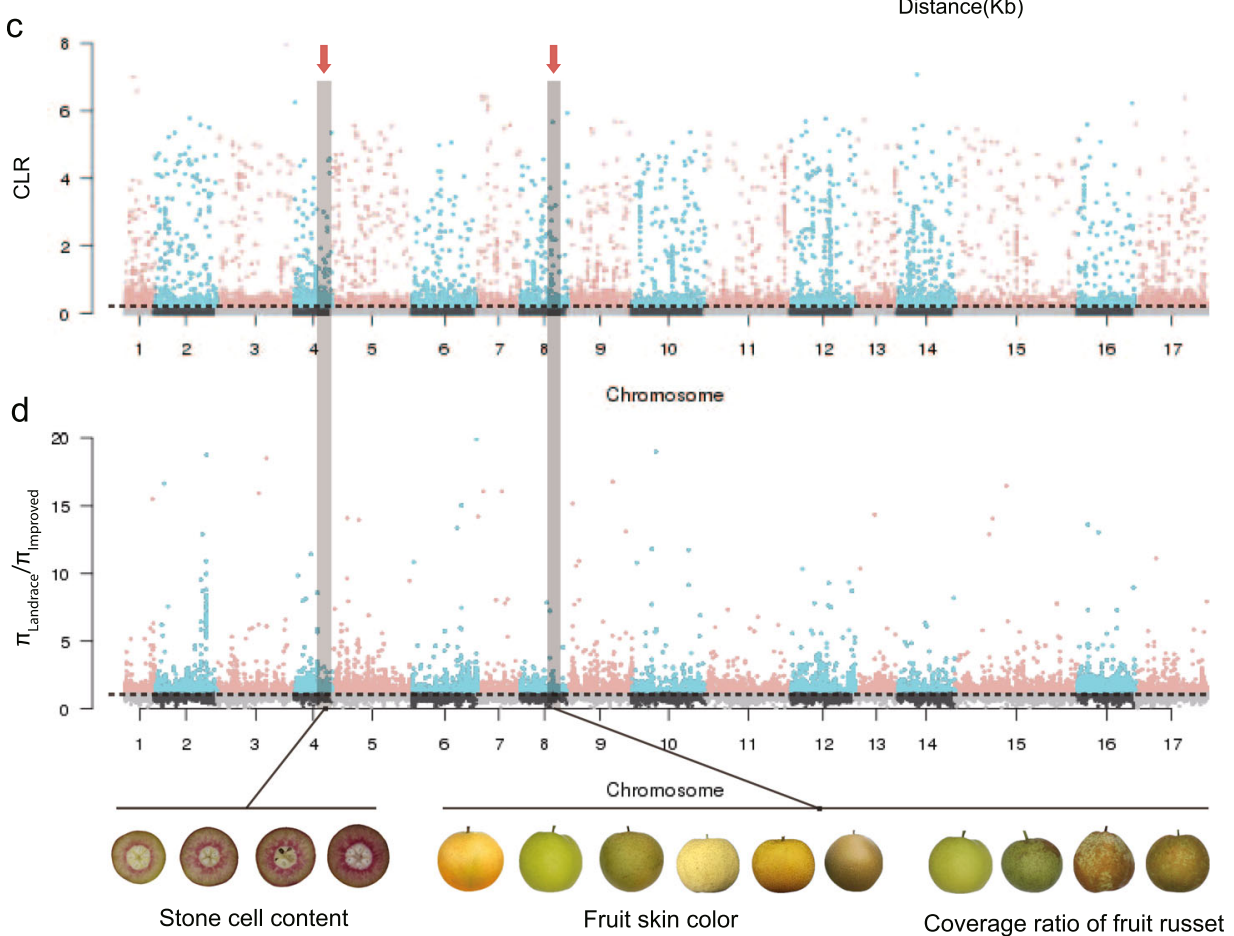

Fig. 2 Diversity and selective sweep of landrace and improved population. a Nucleotide diversity $(\pi)$ of sand pears in landrace $(n=231)$ and improved $(n=$ 81) groups. For each box plot, the lower and upper bounds of the box indicate the first and third quartiles, respectively, and the center line indicates the median. The whisker represents $1.5 \times$ interquartile range of the lower or upper quartile. b LD in the landrace and improved groups. c Selective sweep regions during sand pear improvement (landrace vs improved groups) detected by CLR. The horizontal dashed line refers to the top 5\% threshold of CLR scores. d Selective sweep regions during sand pear improvement (landrace vs improved groups) by $\pi$ ratios. The horizontal dashed line refers to the top $15 \%$ threshold of $\pi_{\text {Landrace }} / \pi_{\text {Improved. }}$. Fruit quality-related traits, stone cell content, coverage ratio of fruit russet, and fruit skin color, are demonstrated in the combined selective sweep regions. 
continuous selection during both domestication and improvement. It was also reported that lignin biosynthesis-related genes were in the selective sweep signals during domestication and improvement at the transcriptome level ${ }^{24}$.

GWAS of fruit quality and phenological traits. A total of 11 agronomic traits were investigated in the 312 sand pears for three consecutive years (Supplementary Data 5 and Supplementary Fig. 4), including three fruit phenological traits (initial bloom period, days of fruit development, and days of vegetative growth) and eight fruit quality traits (single fruit weight, stone cell content, fruit skin color, coverage ratio of fruit russet, location of fruit russet, furrows on fruit surface, direction of carpopodium, and direction of sepal). We identified five candidate loci for the three phenological traits of sand pears and 37 candidate loci for the eight fruit quality traits (Fig. 3a, Supplementary Data 6, 7, and Supplementary Figs. 5-15).

Single fruit weight is very important for fruit production, and our GWAS identified five lead associated SNPs with this trait. Of particular note, one of these candidate SNPs located within a region on chromosome 3 which was previously reported to have a high LOD score for single fruit weight in a QTL study ${ }^{2}$ (Fig. 3b-e). Another candidate SNP (Chr9_2479073) is located within a gene (Pbr001726) encoding a stachyose synthase precursor, suggesting a plausible inference about the impact of this gene on starch accumulation and thus single fruit weight.

Fruit skin color has been a major focus in modern breeding programs for many fruit tree species. Our GWAS results revealed three candidate loci for this trait, and we also found that the skin color-associated loci were in regions under selection during the improvement process for sand pears (Figs. 2c, d and 3a). It was notable that eight of the candidate genes for this trait were annotated as MYB transcription factors, which have been widely reported to regulate fruit skin and flesh colors such as in apple ${ }^{27}$, strawberry $^{28}$, kiwifruit ${ }^{29}$, and tomato ${ }^{30}$.

The furrows on fruit surface affect fruit appearance, but the molecular basis of furrow development remains poorly understood. Our GWAS of furrows on fruit surface trait identified one lead associated SNP on chromosome 13 (Chr13_11586174)
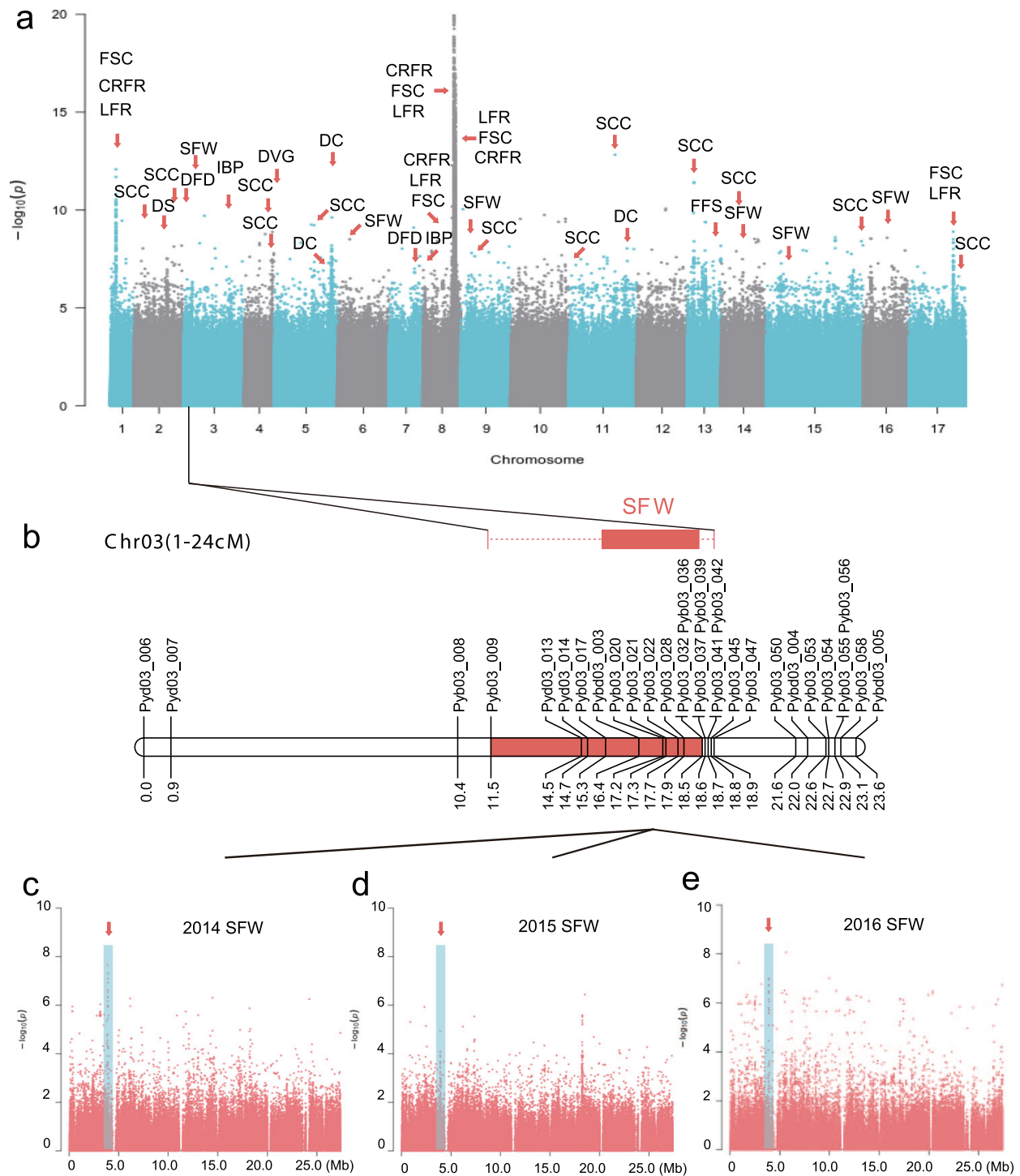

Fig. 3 GWAS of 11 traits in $\mathbf{3 1 2}$ sand pears. a Manhattan plot of GWAS for 11 traits in sand pears. IBP initial bloom period, DFD days of fruit development, DVG days of vegetative growth, FSC fruit skin color, CRFR coverage ratio of fruit russet, LFR location of fruit russet, FFS furrows on fruit surface, DC direction of carpopodium, DS direction of sepal, SFW single fruit weight, SCC stone cell content. $\mathbf{b}$ QTL for trait of single fruit weight in the population of 'Bayuehong' × 'Dangshansuli'. c-e Manhattan plots of chromosome 3 of GWAS for trait of single fruit weight in the years 2014 (c), 2015 (d), and 2016 (e). 
(Fig. 3a and Supplementary Data 7). Furthermore, our GWAS identified one SNP (Chr8_14112990) that was significantly associated with both the coverage ratio of fruit russet and the location of fruit russet, and the associated candidate gene encoded an endoglucanase (Fig. 3a and Supplementary Data 7). The candidate region of coverage ratio of fruit russet and the location of fruit russet was in the selective region, suggesting that these two fruit russet traits could have been under selection during sand pear improvement.

The phenological trait, days of fruit development, dramatically affects fruit maturation, and the appropriate regulation of the pear fruit maturation period could substantially contribute to efficient shipping and storage of pears. Two candidate genome loci associated with this trait were identified, one of them was corresponded to the predicted gene Pbr013897 (Fig. 3a and Supplementary Data 7), which encodes a gibberellin 20-oxidase. It has been reported that gibberellin 20-oxidase is involved in fruit growth in apple ${ }^{31}$, and it could also regulate the flowering and stolon differentiation in strawberry ${ }^{32}$.

A stone cell-related gene from an uncharacterized family. High accumulation of stone cells significantly affects the quality of fruit and is particularly prevalent in sand pear fruit flesh, wherein they distribute in a mosaic pattern ${ }^{33}$. These brachysclereid cells have secondary thickened cell walls and ultimately become parenchyma cells with lignin and cellulose accumulation ${ }^{18}$. Our GWAS identified 12 candidate loci containing a total of 35 SNPs on chromosomes 2, 4, 5, 9, 11, 13, 14, 15, and 17 that were associated with stone cell content (Figs. 3a and 4a, b, and Supplementary Data 7). Since stone cells in pear are mainly produced at early stages of fruit development ${ }^{16}$, we next examined the fruit development RNA-Seq data that we previously generated for four pear cultivars ('Dangshansuli', 'Hosui', 'Yali', and 'Starkrimson') ${ }^{20,34}$ to identify genes in these loci that were highly expressed in the early fruit development. Among the identified candidate genes, in addition to previously characterized lignin regulating genes such as those encoding cinnamate 4-hydroxylases ${ }^{35}$, PbrSTONE (lignin-related stone cell formation; Pbr005042) gene was of particular interest. This gene showed no characterized functions from other organisms, and its encoded protein contained a conserve domain (DUF1223) with an unknown function.

We measured stone cell contents in 312 sand pear accessions, which revealed that the landrace accessions had significantly more stone cells than the improved cultivars $(p$-value $=3.69 \mathrm{E}$ -15) (Supplementary Fig. 16). Genome region of PbrSTONE had high $F_{\mathrm{ST}}$ and reduction of diversity (ROD) values indicated that it has been under selection (Fig. 4a, b). Furthermore, transcript of PbrSTONE was abundantly expressed at early stages of fruit development (Supplementary Fig. 17). We constructed a phylogenetic tree using PbrSTONE and its homologs in 81 eudicot species, and found PbrSTONE and its homologous genes from Malus domestic, Prunus avium, Prunus persica, and Juglans regia clustered in one group (Fig. 4c). We also carried out gene co-expression network analysis to find candidate genes from the lignin biosynthetic pathway and transcription factors correlated with PbrSTONE, and identified seven genes including PbrLAC2 that had similar expression patterns with PbrSTONE (Fig. 4d).

We then conducted a qPCR-based analysis of PbrSTONE gene in different tissues of 'Dangshansuli' plants, which revealed relatively high expression levels of PbrSTONE in anthers, stems, and young fruits (from 21 days after flowering (DAF) to 49 DAF) (Fig. 5a), but much lower levels in pedicels and leaves, as well as in older fruits (after 63 DAF) (Fig. 5a). The expression pattern of PbrSTONE during pear fruit development was consistent with the fact that lignin deposition in stone cells generally occurs during the early stages of fruit development ${ }^{16}$.

To further elucidate the functional role of PbrSTONE in lignin biosynthesis of stone cells, the PbrSTONE overexpression or silencing constructs were agro-infiltrated into 'Dangshansuli' pear fruit at 35 DAF. We confirmed the expression of PbrSTONE (Supplementary Fig. 18a) at 10 days post infiltration, and also observed a dramatic change of the lignin staining signal at the infiltration sites. Overexpressing PbrSTONE significantly increased stone cell and lignin contents, while silencing PbrSTONE decreased them (Fig. 5b-d). The most striking observation from these transient expression experiments was that regulating PbrSTONE expression caused a significant change of the expression of lignin biosynthesis-related genes, particularly the expression of LACs whose homologous genes in Arabidopsis are known to be involved in lignin polymerization ${ }^{36,37}$ (Fig. 5e, f).

We next generated transgenic Arabidopsis lines stably expressing PbrSTONE under the control of the $35 \mathrm{~S}$ promoter. We confirmed the constitutive expression of PbrSTONE in stems of 4week-old plants from three overexpression lines (PbrSTONE-OE) (Supplementary Fig. 18b). In $\mathrm{T}_{3}$ generation homozygous plants, qPCR analysis of 4-week-old wild-type (WT) and PbrSTONE-OE

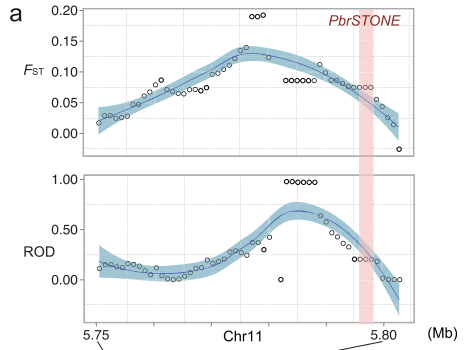

b

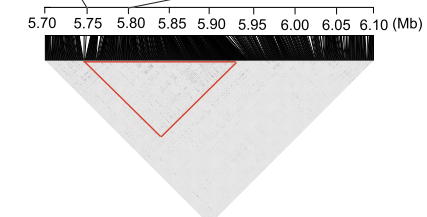

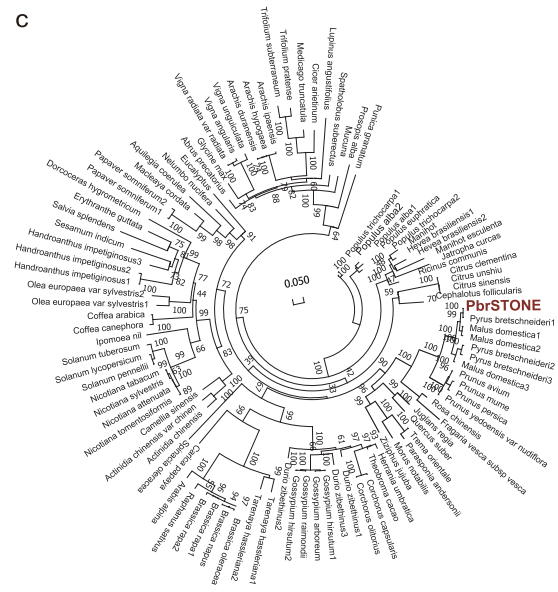

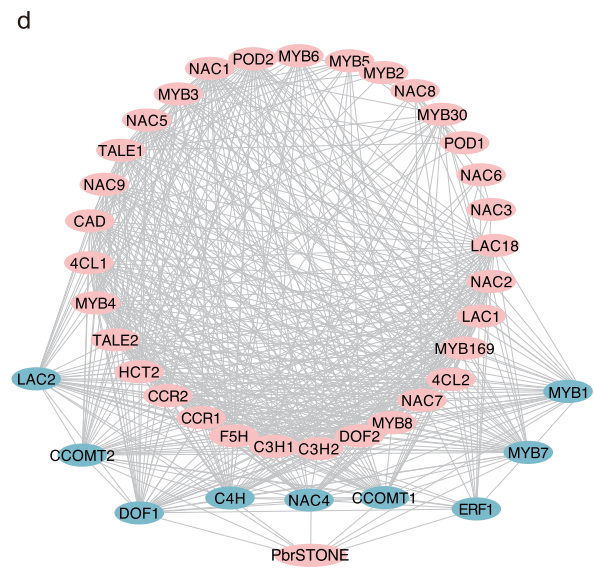

Fig. 4 Stone cell content-related gene PbrSTONE. a Distribution of $F_{\mathrm{ST}}$ and ROD in the PbrSTONE gene region in landrace and improved groups. The shaded region in pink indicates the location of PbrSTONE, and the shade region in blue presents the smooth local regression by 'loess'. $\mathbf{b}$ LD in the

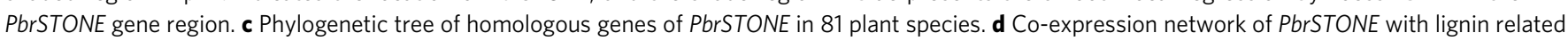
genes. Source data underlying $(\mathbf{c}, \mathbf{d})$ are provided as a Source Data file. 

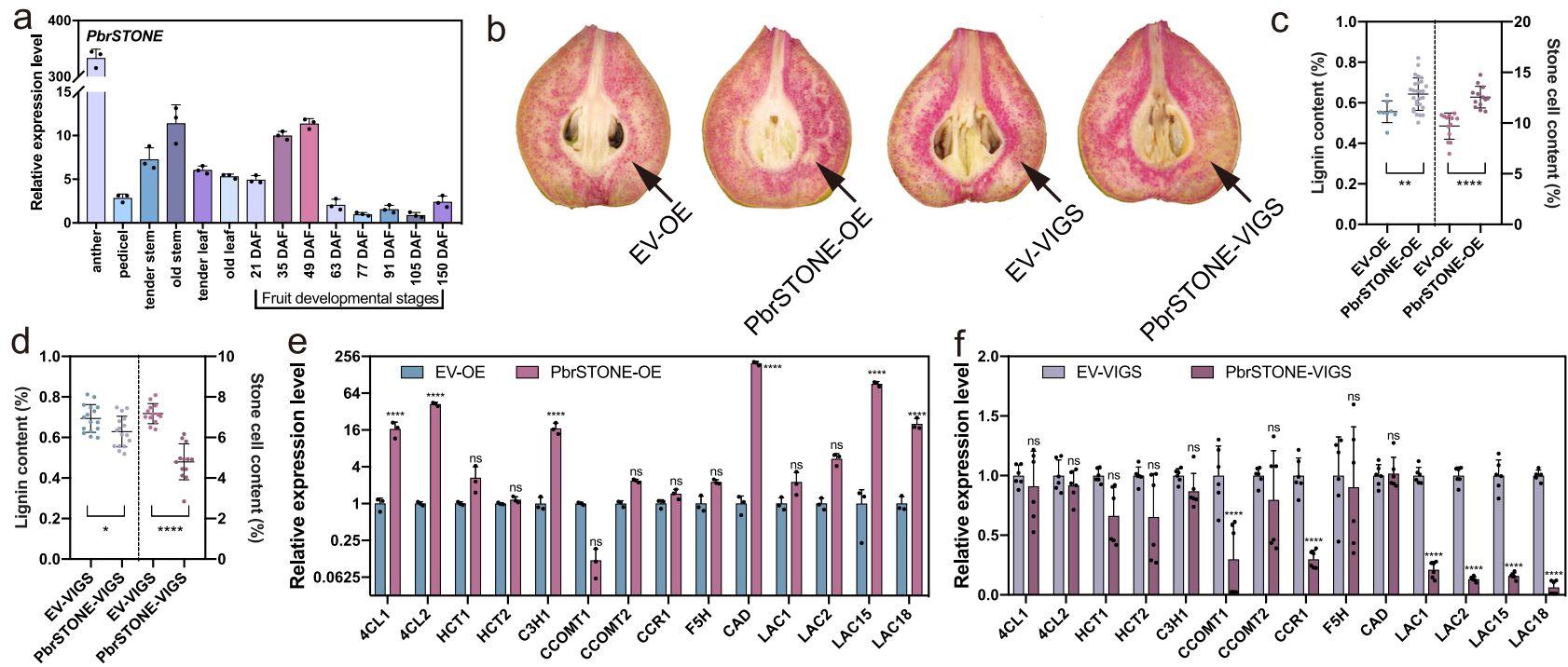

Fig. 5 Functional validation of PbrSTONE in controlling stone cell content and lignin of pears. a Expression profiles of PbrSTONE in different tissues of 'Dangshansuli' plants determined by q-RT-PCR; $n=3$ biologically independent samples. $\mathbf{b}$ Transient assays using PbrSTONE overexpression and silencing constructs in 'Dangshansuli' fruit at 35 days after flowering (DAF). Images were taken at 10 days after agro-infiltration. $\mathbf{c}$, $\mathbf{d}$ Lignin and stone cell contents in the flesh tissue around the infiltration sites. More than six fruits were injected with each construct in an experiment that was repeated three times. EV empty vector, OE overexpression, VIGS virus-induced gene silencing. For lignin content, $n=8,27,16$, and 16 biologically independent samples for EV-OE, PbrSTONEOE, EV-VIGS, and PbrSTONE-VIGS, respectively. For stone cell content, $n=13$ biologically independent samples for all lines. e, $\mathbf{f}$ Expression levels of genes encoding enzymes involved in monolignol biosynthesis and polymerization in the flesh tissue around the infiltration site using qPCR analysis; $n=3$ biologically independent samples for EV-OE and PbrSTONE-OE, and $n=6$ for EV-VIGS and PbrSTONE-VIGS. Data presented are mean \pm SD; $p$-values were determined by two-tailed Student's $t$-test $\left({ }^{\star} p<0.05,{ }^{\star \star} p<0.01,{ }^{\star \star \star} p<0.001,{ }^{\star \star \star \star} p<0.0001\right.$; ns, not significant). Source data are provided as a Source Data file.

plants revealed that the expression of the Arabidopsis homologs of the pear lignin biosynthesis genes $\mathrm{PbrC} 3 \mathrm{H} 1$ (AtC3H1) and PbrLAC15/18 (AtLAC17) were significantly induced by transgenic overexpression of PbrSTONE (Fig. 6a). We analyzed a variety of phenotypes and found several major differences between WT and PbrSTONE-OE plants. Specifically, although the final height and diameter of the primary inflorescence stem of the transgenic lines did not differ from the WT at 8 weeks (Supplementary Fig. 19), the transgenic plants had significantly increased lignin content and decreased root length compared to the WT (Fig. 6b and Supplementary Fig. 19). Moreover, the transgenic plants had significantly increased accumulation of G-lignin monomers compared to WT plants, whereas no differences were observed for the accumulation of S-lignin monomers (Fig. 6c).

Toluidine blue $\mathrm{O}$ staining showed that the PbrSTONE-OE stems contained significantly higher numbers of interfascicular fiber (IF) cell layers than WT plants (Fig. 6d-f). Both the vessels and IF cell tissues of the PbrSTONE-OE lines exhibited stronger chemical staining and auto-fluorescence signals for lignin components than did the WT tissues (Fig. $6 \mathrm{~g}-\mathrm{l}, \mathrm{q}, \mathrm{r}$ ), further supporting that $P b r S T O N E$ expression dramatically alters lignin metabolism. These results support that PbrSTONE functions in both IF formation and lignin deposition in vessels and in IF cell tissues.

To examine anatomical features of the secondary cell walls of the multiple PbrSTONE-OE lines, we conducted transmission electron microscopy (TEM) analysis. We observed that the secondary cell walls of the vessels in PbrSTONE-OE lines were significantly thicker than in WT plants, whereas no such difference in secondary cell wall thickness was observed when examining the IF tissue (Fig. $6 \mathrm{~m}-\mathrm{p}, \mathrm{s}, \mathrm{t}$ ).

In order to better understand how PbrSTONE regulates lignin formation in stone cells, BiFC-LUC assay was conducted with candidate co-expressed genes to identify potential PbrSTONE interacting proteins. PbrSTONE was found to interact with
PbrC3H1 (Fig. 6u), which was positively regulated by a positive regulator of lignification and stone cell development, PbrMYB169 (ref. ${ }^{17}$ ). The homologous gene of $\mathrm{PbrC} 3 \mathrm{H} 1$ in Arabidopsis, AtREF8 (AT2G40890.1), encodes a coumarate 3-hydroxylase $(\mathrm{C} 3 \mathrm{H})$, a $\mathrm{P} 450$-dependent monooxygenase, and is involved in lignin and flavonoid biosynthesis ${ }^{38}$. PbrSTONE was cytoplasm and cytomembrane localized as determined by PbrSTONE-GFP fusion protein in transgenic Arabidopsis root (Supplementary Fig. 20), consistent with the previously reported subcellular localization of $\mathrm{C}^{3} \mathrm{H}^{39}$. Taken together, these results suggested that PbrSTONE may take part in lignification of stone cells via interacting with $\mathrm{PbrC} 3 \mathrm{H} 1$ to form a protein complex.

\section{Methods}

Agronomic trait measurement. All of the measurements for the agronomic traits were based on the Description and data standard for pear (Pyrus spp. ${ }^{40}$. Data were collected in 2014, 2015, and 2016 (some traits were recorded in 2 years because of the unpredictable dropped fruits). Single fruit weight was recorded as the average weight of ten fruits from each sample. Fruit skin color was recorded as ' 1 ' to ' 6 ' according to the Supplementary Fig. 21. Coverage ratio of fruit russet was defined as the ratio of russet area to fruit skin area in the mature fruits. Data were recorded as ' 1 ', ' 3 ', ' 5 ', '7' (Supplementary Fig. 22). Location of fruit russet was recorded as '1', '2', '3', and '4' (Supplementary Fig. 23). Furrows on mature fruit surface were recorded as ' 0 ' and ' 1 ' (Supplementary Fig. 24). Direction of carpopodium was recorded as ' 1 ' and ' 2 ' according to the Supplementary Fig. 25. Direction of sepal was recorded as ' 1 ', '2', and ' 3 ' according to the Supplementary Fig. 26. For days of fruit development, days of vegetative growth, and initial bloom period, data were recorded as the calendar date and were calculated as the number of days from the earliest date to the last one. The stone cell contents were measured according to Tao et al. ${ }^{33}$. Briefly, pulp of mature fruits was collected, stored at $-20^{\circ} \mathrm{C}$ for $24 \mathrm{~h}$, and then homogenated with juice extractor. The sample was centrifuged, and after centrifugation the supernatant was removed. The resulting pellets (stone cells) were re-suspended in water, vortexed, and centrifuged. These washing steps were repeated several times until the supernatant was transparent. The collected stone cells were oven-dried and then weighed three times.

Genome re-sequencing. Young leaves from a total of 312 sand pear accessions (Supplementary Data 1) were collected from the Wuchang Sand Pear Germplasm collection (Hubei Academy of Agricultural Sciences, China). DNA was extracted 
a

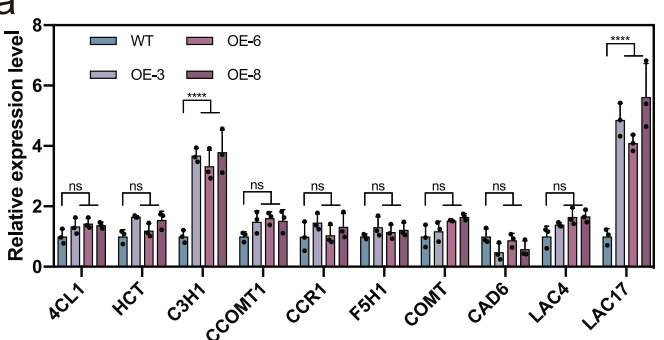

b

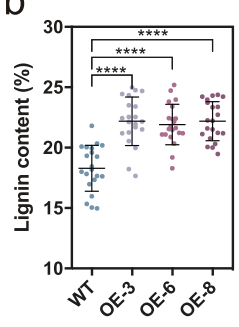

C

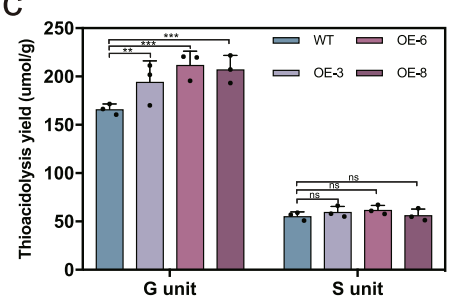

d

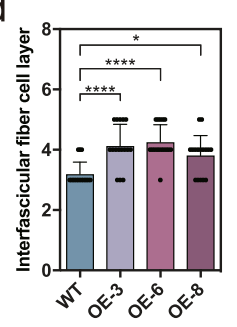

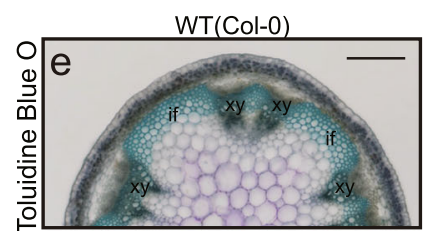
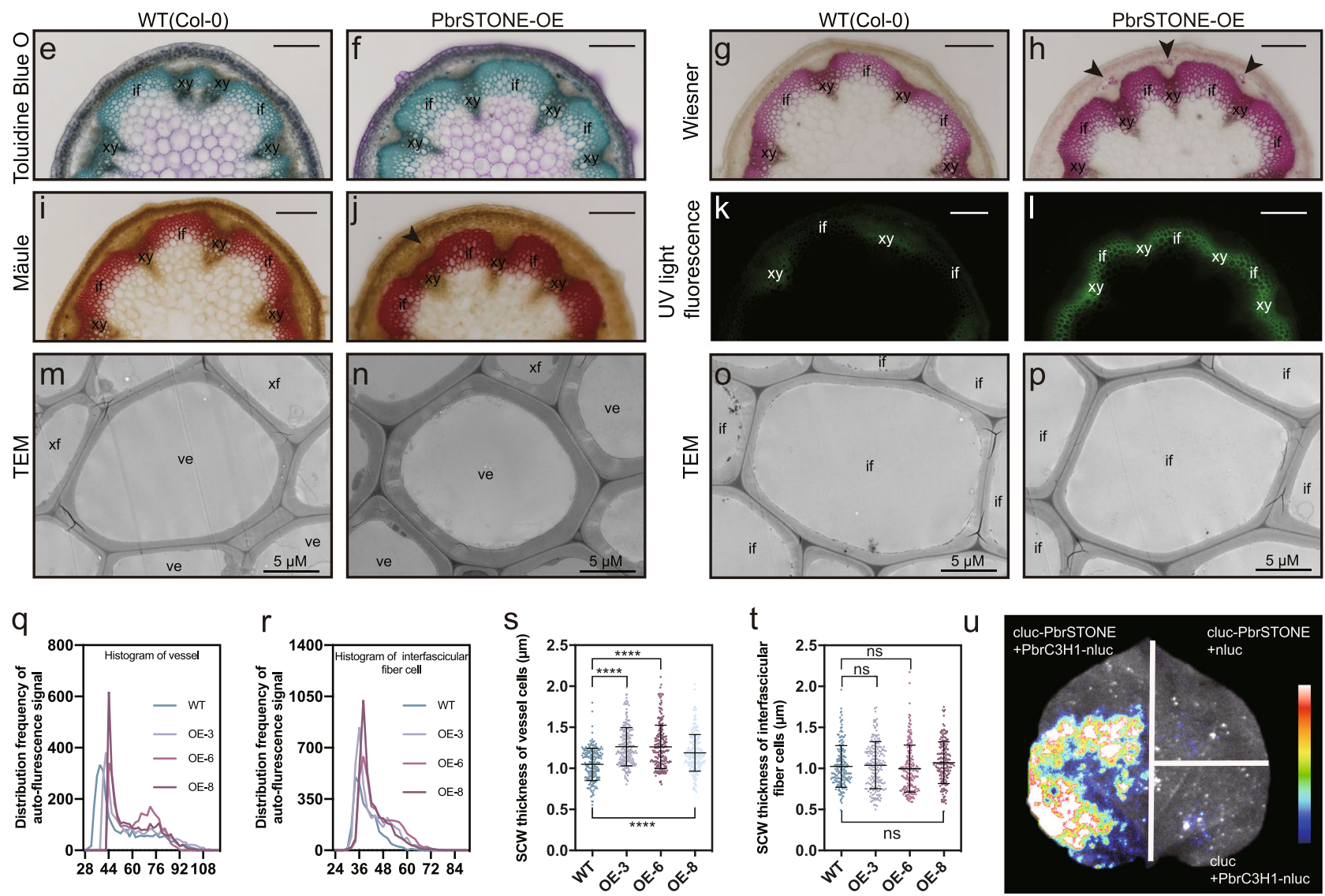

Fig. 6 Functional validation of PbrSTONE in Arabidopsis. a Expression levels of genes encoding enzymes involved in monolignol biosynthesis and polymerization in WT and PbrSTONE transgenic Arabidopsis plants using qPCR analysis; $n=3$ biologically independent samples. b, $\mathbf{c}$ Lignin analysis for WT and PbrSTONE transgenic Arabidopsis plants; $h: n=21$ biologically independent samples for $\mathbf{b}$, and $n=3$ for $\mathbf{c}$. $\mathbf{d}$ Layers of stem interfascicular fiber cells in transgenic Arabidopsis plants expressing PbrSTONE; $n=16$ biologically independent samples. e, $\mathbf{f}$ Cross-sections of 8-week-old inflorescence stems stained with Toluidine blue O. g-j Cross-sections of 8-week-old inflorescence stems stained with Mäule and Wiesner reagent, which bind to S-lignin monomer and G-lignin monomer, respectively. k, I Lignin auto-fluorescence of cross-sections detected under UV light. Scale bars: $200 \mu \mathrm{m}$ in k-r. Bar in k-r = $200 \mu \mathrm{m}$; xy, xylem; if, interfascicular fiber cell. The transgenic and WT plants were cultivated two times and cross-sections were taken from five independent plants which showed similar results. A representative picture from each line is shown. $\mathbf{m}-\mathbf{p}$ Transmission electron micrographs of the cross-section of cells in e; ve, vessel; xf, xylary fiber; if, interfascicular fiber cells. q, $\mathbf{r}$ Statistical analysis of lignin auto-fluorescence signal in the vessel cells and interfascicular fiber cells. More than 12 samples in each line were analyzed. $\mathbf{s}$, $\mathbf{t}$ Statistical analysis of secondary cell wall thickness in the vessel cells and interfascicular fiber cells. More than 50 cells in each line were analyzed. u Firefly luciferase complementation assay in young Nicotiana benthamiana leaves. Data presented are mean \pm SD; $p$-values were determined by two-tailed Student's $t$-test $\left({ }^{\star} p<0.05,{ }^{\star \star} p<0.01,{ }^{\star \star \star} p<0.001,{ }^{\star \star \star \star} p<0.0001\right.$; ns, not significant). Source data underlying (a-t) are provided as a Source Data file.

using plant genomic DNA kits from TIANGEN ${ }^{\oplus}$. DNA quality was checked by NanoPhotometer ${ }^{\oplus}$ spectrophotometer (IMPLEN) and $1 \%$ agarose gels, and DNA concentration was measured by Qubit ${ }^{\circledR}$ DNA Assay Kit in a Qubit 2.0 Flurometer (Life Technologies). NEB Next Ultra DNA Library Prep Kit for Illumina (NEB) was used to generate the sequencing libraries. The cBot Cluster Generation System of the HiSeq 4000 PE Cluster Kit (Illumina) was used for clustering of the indexcoded libraries, and the Illumina HiSeq 4000 system was used to sequence the DNA libraries (150 bp paired-end reads).

Data filtering and SNP calling. Quality of the 150-bp paired-end reads was checked by FastQC (http://www.bioinformatics.babraham.ac.uk/projects/fastqc/), and the reads were then processed using Trimmomatic ${ }^{41}$ to trim the adapters and sequences with average quality per base below 20 . The trimmed reads with length of at least $40 \mathrm{bp}$ were mapped against the reference pear genome ${ }^{20}(P$. bretschneideri) using BWA aln ${ }^{42}$ with parameters ' $-\mathrm{n} 0.04$-o 1 -e 2'. Genome Analysis Toolkit (4.1.4) was used for SNP calling with unified genotyper method ${ }^{43}$, and the SNPs were filtered using criteria of MAF $\geq 0.03$ and missing rate no more than $30 \%$.

$\boldsymbol{F}_{\mathrm{ST}}$ and ROD analysis and selective sweep detection. $F_{\mathrm{ST}}$ and ROD were calculated using vcftools ${ }^{44}$ with a window size of $10 \mathrm{~kb}$ and a window step size of $5 \mathrm{~kb}$, and 'gplots' (https://cran.r-project.org/web/packages/gplots) in the R packages was applied for presentation. RNA-Seq data of landrace and improved pears ${ }^{24}$ were 
used for PbrSTONE selection analysis. Candidate selective sweeps were first detected using the SweeD's composite likelihood rate (CLR) test of the site frequency spectra ${ }^{45}$ with a window size of $10 \mathrm{~kb}$, windows with top $5 \%$ CLR scores were considered candidate regions under a selective sweep. These regions were further filtered using $\pi$ ratios, and only those having top $15 \% \pi$ ratios were considered the final selective sweeps. GWAS associated loci within $550 \mathrm{bp}$ up or downstream of the identified selective sweeps were extracted. We also extracted genes in the selective sweep regions based on the gene annotation of the 'Dangshansuli' genome covered by sweeps.

Genome-wide association studies. The pear population structure was calculated by STRUCTURE ${ }^{46}$ with the simulated population structure (K value) ranging from 2 to 6. GWAS were performed using both TASSEL ${ }^{47}$ and EMMAX programs ${ }^{48}$. Candidate associated loci identified by both TASSEL and EMMAX were considered further in our study, as well as those identified in one program but with genes annotated as known to be related to the corresponding traits. TASSEL was also used for the phylogenetic tree (Neighbor-Joining method), PCA, kinship matrix of the population, and LD analyses. General linear model (GLM) and mixed linear model (MLM) used for GWAS analyses were compared based on the Q-Q plots, and we finally found MLM-PCA-kinship was the best for our sand pear population. The $R^{2}$ of $\mathrm{LD}$ was visualized using the LDheatmap $\mathrm{R}$ package ${ }^{49}$. The cutoff was used as $P=1 \mathrm{E}-5$. The genomic inflation factor was calculated by $\mathrm{R}$ package of GenABEL ${ }^{50}$ with regression method.

Gene annotation and statistical analysis. We used the annotated information of pear genes from KEGG and Swissport database. Specifically, the DNA and amino acid sequences of PbrSTONE were used for BLAST analysis against the NCBI nucleotide, protein and conserved domain databases. We also used hidden Markov model analysis to compare PrbSTONE amino acid sequences to the Pfam domain database ${ }^{51}$. We used Student's $t$-tests or one-way ANOVA to compare statistical differences using GraphPad prism 8.0 software (GraphPad Software).

Gene co-expression analysis and phylogeny of PbrSTONE. Genes (4CL1, 4CL2, C3H1, C3H2, C4H, CAD, CCOMT1, CCOMT2, CCR1, CCR2, F5H, HCT1, HCT2, $P O D 1, P O D 2, L A C 1, L A C 2$, and $L A C 18)$ from lignin biosynthetic pathway and transcription factors (LAC1, LAC2, LAC18, MYB1-8, MYB24, MYB30, MYB169, NAC1-9, ERF1, DOF1, DOF2, TALE1, and TALE2) were selected as the candidate genes for co-expression network analysis with PbrSTONE using WGCNA ${ }^{52}$, using the fruit gene expression data of six developmental stages of five pear subspecies from our previous study ${ }^{35}$. The correlation relationship was visualized using Cytoscape (http://www.cytoscape.org).

We performed 'blastp' of the PbrSTONE protein sequence against the NCBI nr database, and obtained 99 proteins from 81 eudicot species with an e-value $<1 \mathrm{E}-5$ and sequence identity above $60 \%$. All of the identified proteins were aligned with CLUSTAL $^{53}$ (version 2.1), and the Maximum Likelihood method in IQ-TREE ${ }^{54}$ (multicore version 1.6.10) was applied for the phylogenic tree construction with 1000 bootstraps.

Gene expression analysis using $\mathbf{\text { qPCR}}$. cDNA was synthesized using one-step gDNA removal and cDNA synthesis kit (Transgen). qPCR was performed using the LightCycler 480 SYBR GREEN Master system (Roche). Primers are shown in Supplementary Data 8 . The analysis was conducted using three biological and three technical repeats. Relative expression levels of each gene were calculated using the 2 $-\triangle \triangle \mathrm{CP}$ method. PbrGAPDH and Atactin/AtEF1 $\alpha$ were used as reference genes for pear and Arabidopsis, respectively.

\section{Transient transformation of PbrSTONE in pear fruit flesh. To transiently} overexpress PbrSTONE, the full-length coding sequence of PbrSTONE was fused in frame to the N-terminus of GFP under the control of CaMV $35 \mathrm{~S}$ promoter in the binary vector pCAMBIA1302 (p1302) to form the fusion vector 35S::PbrSTONEGFP. For TRV virus-induced gene silencing (VIGS), the partial coding sequences of PbrSTONE (1-276 bp) was amplified using primers listed in Supplementary Data 8 and the amplified fragment was inserted into the vector TRV2. The fusion constructs and the control vector (p1300) were transferred into Agrobacterium tumefaciens strain GV3101 by the freeze-thaw method. For transiently transformed pear fruit flesh, the cells were infiltrated into 'Dangshansuli' fruit flesh at 35 DAF using needleless syringes. The transformed fruits were placed in the dark at $22{ }^{\circ} \mathrm{C}$ overnight and then incubated in a growth chamber at $22^{\circ} \mathrm{C}$ under $16 \mathrm{~h}$ photoperiod for 10 days before being examined and imaged ${ }^{16}$. Six fruits were injected with each construct in an experiment that was repeated three times independently. The acetyl bromide-based method was used to detect lignin in fruit flesh tissue. The lignin content was shown as a percentage (calculated lignin content/ dry weight of stone cells $\times 100 \%)^{16}$.

Arabidopsis transformation. Agrobacterium tumefaciens containing the vector 35S::PbrSTONE-GFP were transformed into Arabidopsis Col-0 plants using using the floral dip method ${ }^{55}$. Expression levels of PbrSTONE in primary inflorescence stem of two-week-old $\mathrm{T}_{1}$ plants were analyzed by semi-quantitative RT-PCR using primers listed in Supplementary Data 8. Relatively high levels of PbrSTONE expression were measured in three transgenic lines (OE-3, OE-6, and OE-8), which were selected to generate $T_{3}$ homozygous plants for the subsequent analyses. Root length of each transgenic line and the WT control were measured at 7 days after germination on agar medium. Subsequently the plants were transplanted in a glasshouse with a $16 \mathrm{~h}$ photoperiod. The height and diameter of primary inflorescence stem were determined for each transgenic line and the WT control at 8 weeks post germination.

Lignin analysis. For WT and $\mathrm{T}_{3}$ transgenic Arabidopsis plants, the basal $10 \mathrm{~cm}$ of the primary inflorescence stem was chopped and pooled together from five plants. Samples of stem pieces were extracted sequentially for water, ethanol, chloroform, and acetone to gain purified cell wall residue, then dried for the subsequent lignin analysis. Acetyl bromide-soluble lignin content was estimated according to a standard procedure ${ }^{56}$. The lignin composition was determined by thioacidolysis ${ }^{57}$. The released lignin monomers were derivatized with $\mathrm{N}, \mathrm{O}$-bis (trimethylsilyl) acetamide and quantified by GC-MS as their trimethylsilylated derivatives. The analytical data were presented as the mean values obtained from the same amount of starting material harvested from the pooled xylem tissues of five plants.

Microscopy. For light microscopy analysis, the basal $1 \mathrm{~cm}$ of primary inflorescence stems of 8-week-old Arabidopsis WT and PbrSTONE transgenic plants were harvested and fixed in formalin-acetic acid-alcohol fixative (1.9\% formaldehyde solution [v/v], 63\% ethyl alcohol [v/v], 5\% glacial acetic acid [v/v], 30.1\% water [v/ v]) at $4{ }^{\circ} \mathrm{C}$ for 7 days. Cross sections (100 $\mu \mathrm{m}$ thickness) of inflorescence stems were sectioned with a Leica VT1000S vibratome (Leica Mikrosysteme, Germany), stained with Toluidine Blue $\mathrm{O}$, Mäule and Wiesner reagent separately, and then observed with Nikon Ni-U microscope (Nikon, Japan) ${ }^{58}$. Nikon Ti-E microscope (Nikon, Japan) was used to visualize the lignin auto-fluorescence under UV light (excitation at 355/25). Fluorescence intensity was measured on the images with Image software.

TEM analyses were performed with the stem samples that were fixed in a fixative $(2.5 \%[\mathrm{v} / \mathrm{v}]$ glutaraldehyde, $50 \mathrm{~mm}$ sodium phosphate $[\mathrm{pH} 7.2]$, and $4 \%[\mathrm{v} /$ $\mathrm{v}$ ] formaldehyde) for $12 \mathrm{~h}$ and further fixed by $0.5 \%$ osmic acid ${ }^{59}$. The tissues were then dehydrated and embedded in LR white resin (Polysciences, Warrington, PA, USA). Ultrathin sections were stained with uranyl acetate and lead citrate. The secondary wall thickness of vessel cells was gained on the images of TEM with ImageJ.

Bimolecular fluorescence complementation analysis. pCAMBIA1300-NLuc and pCAMBIA1300-CLuc were used for bimolecular fluorescence complementation (BiFC) analysis ${ }^{60}$. Full-length coding sequences of $\mathrm{PbrSTONE}$ and $\mathrm{PbrC} 3 \mathrm{H} 1$ were amplified using the primers listed in Supplementary Data 8 and then inserted individually into the two plasmids and transferred into A. tumefaciens strains GV3101. Equal amounts of PbrSTONE and $\mathrm{PbrC3H1}$ combinations were coinfiltrated into the young leaves of Nicotiana benthamiana plants. On the second day post-infiltration, $1 \mathrm{mM}$ luciferin was sprayed onto the leaves, and the samples were incubated in the dark for 5-10 min. LUC images were captured using a low light cooled CCD imaging apparatus (Tanon 5200 Multi).

Reporting summary. Further information on research design is available in the Nature Research Reporting Summary linked to this article.

\section{Data availability}

Data supporting the findings of this work are available within the paper and its Supplementary Information files. A reporting summary for this Article is available as a Supplementary Information file. The datasets and plant materials generated and analyzed during the current study are available from the corresponding author upon request. Raw genome resequencing reads have been deposited into the NCBI sequence read archive (SRA) under BioProject accession PRJNA563813. Sequence data of PbrSTONE and $\mathrm{PbrC} 3 \mathrm{H} 1$ can be found at GenBank with the accession numbers MT711883 and MT711884, respectively. SNPs identified in this study are available at Zenodo (https:// zenodo.org/deposit/3971245). Figures 1, 2, 3a, 3c, 3d, 3e and $4 b$ are generated from the SNPs data using parameters specified in 'Methods'. Source data are provided with this paper.

Received: 9 September 2019; Accepted: 25 January 2021; Published online: 18 February 2021

\section{References}

1. Rom, R. C. \& Robert, F. C. Rootstocks for Fruit Crops (Wiley, 1987).

2. Wu, J. et al. High-density genetic linkage map construction and identification of fruit-related QTLs in pear using SNP and SSR markers. J. Exp. Bot. 65, 5771-5781 (2014). 
3. Cho, K. H. et al. Development of AFLP and CAPS markers linked to the scab resistance gene, Rvn2, in an inter-specific hybrid pear (Pyrus spp.). J. Hortic. Sci. Biotechnol. 84, 619-624 (2009).

4. Dondini, L. et al. Identifying QTLs for fire-blight resistance via a European pear (Pyrus communis L.) genetic linkage map. Mol. Breed. 14, 407-418 (2004).

5. PMF, Le. Roux et al. Redefinition of the map position and validation of a major quantitative trait locus for fire blight resistance of the pear cultivar 'Harrow Sweet' (Pyrus communis L.). Plant Breed. 131, 656-664 (2012).

6. Yamamoto, T. et al. Identification of QTLs controlling harvest time and fruit skin color in Japanese pear (Pyrus pyrifolia Nakai). Breed. Sci. 64, 351-361 (2014).

7. Thornsberry, J. M. et al. Dwarf8 polymorphisms associate with variation in flowering time. Nat. Genet. 28, 286-289 (2001).

8. Huang, X. H. et al. Genome-wide association studies of 14 agronomic traits in rice landraces. Nat. Genet. 42, 961-976 (2010).

9. Yano, K. et al. Genome-wide association study using whole-genome sequencing rapidly identifies new genes influencing agronomic traits in rice. Nat. Genet. 48, 927-934 (2016).

10. Cao, K. et al. Genome-wide association study of 12 agronomic traits in peach. Nat. Commun. 7, 13246 (2016).

11. Mariette, S. et al. Genome-wide association links candidate genes to resistance to Plum Pox Virus in apricot (Prunus armeniaca). N. Phytol. 209, 773-784 (2016).

12. Li, X. et al. Development of an integrated $200 \mathrm{~K}$ SNP genotyping array and application for genetic mapping, genome assembly improvement and genome wide association studies in pear (Pyrus). Plant Biotechnol. J. 17, 1582-1594 (2019).

13. Yao, G. F. et al. Map-based cloning of the pear gene MYB114 identifies an interaction with other transcription factors to coordinately regulate fruit anthocyanin biosynthesis. Plant J. 92, 437-451 (2017).

14. Li, J. M. et al. A new insight into the evolution and functional divergence of SWEET transporters in Chinese white pear (Pyrus bretschneideri). Plant Cell Physiol. 58, 839-850 (2017).

15. Xu, L. L., Qiao, X., Zhang, M. Y. \& Zhang, S. L. Genome-wide analysis of aluminum-activated malate transporter family genes in six rosaceae species, and expression analysis and functional characterization on malate accumulation in Chinese white pear. Plant Sci. 274, 451-465 (2018).

16. Xue, C. et al. PbrmiR397a regulates lignification during stone cell development in pear fruit. Plant Biotechnol. J. 17, 103-117 (2019).

17. Xue, C. et al. PbrMYB169 positively regulates lignification of stone cells in pear fruit. J. Exp. Bot. 70, 1801-1814 (2019).

18. Smith, W. W. The course of stone cell formation in pear fruits. Plant Physiol. 10, 587-611 (1935)

19. Cai, Y. P. et al. Study of the structure and biosynthetic pathway of lignin in stone cells of pear. Sci. Hortic. 125, 374-379 (2010).

20. Wu, J. et al. The genome of the pear (Pyrus bretschneideri Rehd.). Genome Res. 23, 396-408 (2013).

21. Wu, J. et al. Diversification and independent domestication of Asian and European pears. Genome Biol. 19, 77 (2018).

22. Zhou, Z. et al. Resequencing 302 wild and cultivated accessions identifies genes related to domestication and improvement in soybean. Nat. Biotechnol. 33, 408-414 (2015).

23. $\mathrm{Xu}, \mathrm{X}$. et al. Resequencing 50 accessions of cultivated and wild rice yields markers for identifying agronomically important genes. Nat. Biotechnol. 30, 105-111 (2012).

24. Li, X. L. et al. Comparative transcriptomic analysis provides insight into the domestication and improvement of pear (P. pyrifolia) fruit. Plant Physiol. 180, 435-452 (2019).

25. Lam, H. M. et al. Resequencing of 31 wild and cultivated soybean genomes identifies patterns of genetic diversity and selection. Nat. Genet. 42, 1053-1059 (2010).

26. Duan, N. B. et al. Genome re-sequencing reveals the history of apple and supports a two-stage model for fruit enlargement. Nat. Commun. 8, 249 (2017).

27. An, J. P. et al. R2R3-MYB transcription factor MdMYB23 is involved in the cold tolerance and proanthocyanidin accumulation in apple. Plant J. 96, 562-577 (2018).

28. Schaart, J. G. et al. Identification and characterization of MYB-bHLH-WD40 regulatory complexes controlling proanthocyanidin biosynthesis in strawberry (Fragaria $x$ ananassa) fruits. New Phytol. 197, 454-467 (2013).

29. Ampomah-Dwamena, C. et al. A kiwifruit (Actinidia deliciosa) R2R3-MYB transcription factor modulates chlorophyll and carotenoid accumulation. New Phytol. 221, 309-325 (2019).

30. Dal Cin, V. et al. Identification of genes in the phenylalanine metabolic pathway by ectopic expression of a MYB transcription factor in tomato fruit. Plant Cell 23, 2738-2753 (2011).

31. Kusaba, S. et al. Characterization of gibberellin 20-oxidase gene in apple. Acta Hortic. 538, 605-608 (2000).
32. Tenreira, T. et al. A specific gibberellin 20-oxidase dictates the floweringrunnering decision in diploid strawberry. Plant Cell 29, 2168-2182 (2017).

33. Tao, S. T. et al. Anatomy, ultrastructure and lignin distribution of stone cells in two Pyrus species. Plant Sci. 176, 413-419 (2009).

34. Zhang, M. Y. et al. Distinct transcriptome profiles reveal gene expression patterns during fruit development and maturation in five main cultivated species of pear (Pyrus L.). Sci. Rep. 6, 28130 (2016).

35. Lewis, A. N. G. et al. Trends in lignin modification: a comprehensive analysis of the effects of genetic manipulations/mutations on lignification and vascular integrity. Phytochemistry 61, 221-294 (2002).

36. Berthet, S. et al. Disruption of LACCASE4 and 17 results in tissue-specific alterations to lignification of Arabidopsis thaliana stems. Plant Cell 23, 1124-1137 (2011).

37. Zhao, Q. et al. LACCASE is necessary and nonredundant with PEROXIDASE for lignin polymerization during vascular development in Arabidopsis. Plant Cell 25, 3976-3987 (2013)

38. Franke, R. et al. Changes in secondary metabolism and deposition of an unusual lignin in the ref8 mutant of Arabidopsis. Plant J. 30, 47-59 (2002).

39. Fraser, C. M. \& Chapple, C. The phenylpropanoid pathway in Arabidopsis. Arabidopsis Book 9, e0152 (2011).

40. Cao, Y. -F., Liu, F., Hu, H. \& Zhang, B. Description and Data Standard for Pear (Pyrus spp.) (China Agriculture Press, 2006).

41. Bolger, A. M., Lohse, M. \& Usadel, B. Trimmomatic: a flexible trimmer for Illumina sequence data. Bioinformatics 30, 2114-2120 (2014).

42. $\mathrm{Li}, \mathrm{H}$. et al. Fast and accurate short read alignment with Burrows-Wheeler transform. Bioinformatics 25, 1754-1760 (2009).

43. McKenna, A. et al. The Genome Analysis Toolkit: a MapReduce framework for analyzing next-generation DNA sequencing data. Genome Res. 20, 1297-1303 (2010)

44. Danecek, P. et al. The variant call format and VCFtools. Bioinformatics 27, 2156-2158 (2011)

45. Pavlidis, P., Zivkovic, D., Stamatakis, A. \& Alachiotis, N. SweeD: likelihoodbased detection of selective sweeps in thousands of genomes. Mol. Biol. Evol. 30, 2224-2234 (2013).

46. Pritchard, J. K. et al. Inference of population structure using multilocus genotype data. Genetics 155, 945-959 (2000).

47. Zhang, Z. W. et al. Mixed linear model approach adapted for genome-wide association studies. Nat. Genet. 42, 355-360 (2010).

48. Kang, H. M. et al. Variance component model to account for sample structure in genome-wide association studies. Nat. Genet. 42, 348-354 (2010).

49. Ji-Hyung Shin, S. B. et al. LDheatmap: graphical display of pairwise linkage disequilibria between SNPs. J. Stat. Softw. 16, Code Snippet 3 (2006).

50. Aulchenko, Y. S. et al. Genome-wide rapid association using mixed model and regression: a fast and simple method for genome-wide pedigree-based quantitative trait loci association analysis. Genetics 177, 577-585 (2007).

51. El-Gebali, S. et al. The Pfam protein families database in 2019. Nucleic Acids Res. 47, 427-432 (2019).

52. Langfelder, P. \& Horvath, S. WGCNA: an R package for weighted correlation network analysis. BMC Bioinformatics 9, 559 (2008)

53. Larkin, M. A. et al. Clustal W and Clustal X version 2.0. Bioinformatics 23, 2947-2948 (2007)

54. Chernomor, O., von Haeseler, A. \& Minh, B. Q. Terrace aware data structure for phylogenomic inference from supermatrices. Syst. Biol. 65, 997-1008 (2016).

55. Clough, S. J. \& Bent, A. F. Floral dip: a simplified method for Agrobacteriummediated transformation of Arabidopsis thaliana. Plant J. 16, 735-743 (1998)

56. Acker, R. V. et al. Lignin biosynthesis perturbations affect secondary cell wall composition and saccharification yield in Arabidopsis thaliana. Biotechnol. Biofuels 6, 1-17 (2013).

57. Lapierre, C., Pollet, B. \& Rolando, C. New insights into the molecular architecture of hardwood lignins by chemical degradative methods. Res. Chem. Intermediat. 21, 397-412 (1995).

58. Mitra, P. P. \& Loque, D. Histochemical staining of Arabidopsis thaliana secondary cell wall elements. J. Vis. Exp. e51381 (2014).

59. Vanholme, R. et al. Caffeoyl shikimate esterase (CSE) is an enzyme in the lignin biosynthetic pathway in Arabidopsis. Science 341, 1103-1106 (2013).

60. Chen, H. et al. Firefly luciferase complementation imaging assay for proteinprotein interactions in plants. Plant Physiol. 146, 368-376 (2008).

\section{Acknowledgements}

This work was supported by the National Key Research and Development Program (2018YFD1000200), the National Science Foundation of China (31725024, 31901978,31801835, 31901983), the Earmarked Fund for the China Agriculture Research System (CARS-28), the Earmarked Fund for Jiangsu Agricultural Industry Technology System JATS[2020]401, the Natural Science Foundation of Jiangsu Province

(SBK2018043235), the China Postdoctoral Science Foundation (2018M630565), and the US National Science Foundation (IOS-1855585). We thank Qingwen Liu, Lun Liu, 
Jiaying Zhang for providing pear photos and other contributions to this work. This project was supported by the Bioinformatics Center of Nanjing Agricultural University.

\section{Author contributions}

J.W., S.Z., and Z.F. designed the project. M.Y.Z. performed the data analysis. C.X. and Y.X. performed the functional gene verification with assistance from X.C.. H.H., J.F., M.Q. and B.B. collected the phenotype data. J.L., R.W., C.Z., S.T., C.G., X.L., S.W., G.Y., Y.L., and M.S. gave suggestions of the analysis. M.Y.Z., C.X., and J.W. wrote and revised the paper. Z.F. provided guidance on data analysis, and revised the manuscript.

\section{Competing interests}

The authors declare no competing interests.

\section{Additional information}

Supplementary information The online version contains supplementary material available at https://doi.org/10.1038/s41467-021-21378-y.

Correspondence and requests for materials should be addressed to Z.F., S.Z. or J.W.

Peer review information Nature Communications thanks Pere Arús, and the other, anonymous, reviewer(s) for their contribution to the peer review of this work.
Reprints and permission information is available at http://www.nature.com/reprints Publisher's note Springer Nature remains neutral with regard to jurisdictional claims in published maps and institutional affiliations.

(c) (i) Open Access This article is licensed under a Creative Commons Attribution 4.0 International License, which permits use, sharing, adaptation, distribution and reproduction in any medium or format, as long as you give appropriate credit to the original author(s) and the source, provide a link to the Creative Commons license, and indicate if changes were made. The images or other third party material in this article are included in the article's Creative Commons license, unless indicated otherwise in a credit line to the material. If material is not included in the article's Creative Commons license and your intended use is not permitted by statutory regulation or exceeds the permitted use, you will need to obtain permission directly from the copyright holder. To view a copy of this license, visit http://creativecommons.org/ licenses/by/4.0/.

(C) The Author(s) 2021 